https://helda.helsinki.fi

\title{
Inhibitory control - Important trait for explosive detection performance in police dogs?
}

\section{Tiira, Katriina}

2020-03

Tiira , K , Tikkanen , A \& Vainio , O 2020 , ' Inhibitory control - Important trait for explosive detection performance in police dogs? ' , Applied Animal Behaviour Science , vol. 224 , 104942 . https://doi.org/10.1016/j.applanim.2020.104942

http://hdl.handle.net/10138/327715

https://doi.org/10.1016/j.applanim.2020.104942

cc_by_nc_nd

acceptedVersion

Downloaded from Helda, University of Helsinki institutional repository.

This is an electronic reprint of the original article.

This reprint may differ from the original in pagination and typographic detail.

Please cite the original version. 


\section{Inhibitory control - important trait for explosive 2 detection performance in police dogs?

4 Katriina Tiira ${ }^{1,2}$, Antti Tikkanen ${ }^{3}$ \& Outi Vainio ${ }^{2}$

$5 \quad{ }^{1}$ SmartDOG, Pietilänkatu 5, 11130 Riihimäki, Finland

${ }^{2}$ Department of Equine and Small Animal Medicine, P.O. Box 57, FI-00014 University of Helsinki,

7 Finland

${ }^{3}$ Police Dog Training Centre, Koirakoulunkatu, FI-13130 Hämeenlinna, Finland

Corresponding author: Katriina Tiira, Katriina.tiira@helsinki.fi

Keywords: cognition, dog, inhibitory control, motor inhibition, explosive detection, working dog

\section{Abstract}

Working dogs are used for a range of important operational tasks. However, there is little knowledge on the possible association between cognitive traits and the actual working dog performance. This study investigated whether motor inhibition, persistence, problem-solving strategies, and spatial problem-solving are associated with explosive detection success in specially trained police dogs. Dogs $(N=24)$ were tested with a cognitive test battery, and subsequently they participated in an explosive detection test. The explosive searching situation and the location of the test was such that it would reflect as much as possible a real-life situation. Canine handlers also filled in a questionnaire regarding their dog's working behaviour. We found that those dogs that were more successful in explosive detection task had better motor inhibition in a cylinder task compared to dogs with lower success in an explosive search task. Furthermore, we found that dogs that made more errors in the cylinder task were generally more likely to give up searching sooner, as reported by their handlers, 
25 and also abandon sooner the problem-solving task in behavioural test. This study suggests that 26 inhibitory control, specifically motor inhibition, may be an important aspect to consider when 27 selecting suitable dogs for explosive detection tasks.

28

29

30

31

32 


\section{Introduction}

Stable individual differences in cognitive abilities are found in humans (Corr, 2010), and have also been documented in nonhuman animals in recent years (Corr, 2010; Griffin et al., 2015; Brucks et al., 2017). Cognitive abilities, such as behavioural inhibition, associative learning, problem-solving, and attention are important traits for academic success in humans (Moffitt et al., 2011), but little is known of the consequences of cognitive variation for canine working success. Several studies have focused on identifying potentially successful working dogs as early as possible because all forms of dog training (e.g. military, guide, police, assistance dog, drug detection, explosive detection) is costly (Wilsson and Sundgren, 1998; Sinn et al., 2010; Tomkins et al., 2011). At the moment rejection rates in training programmes can be as high as $46-50 \%$ (Ennik et al., 2006). The majority of studies, and personality test batteries used in the selection process for working dog training, focus on testing personality traits such as shyness - boldness, trainability, activity, aggression and sociability. The significance of cognitive traits in dogs in relation to working dog success has been assessed, to our knowledge, only in two studies. In guide dogs, the authors found that both the personality (temperament) and the problem-solving traits were important in determining the success in guide dog program (Bray et al., 2017). In a more recent study, the likelihood of engaging in eye contact with the experimenter was found to be important for the success in assistance dog (MacLean. and Hare, 2018) . For the explosive search dogs, instead, the researchers found that traits such as a short-term memory and the sensitivity to human communicative signals were important (MacLean and Hare, 2018). However, in their study, their assessment of the "success" in explosive search was based on the trainer's opinion from these dogs from the training period. In this study, instead, we used a measure of success, which reflects closely a real explosive search situation. We also developed a short cognitive test battery (approximately $20 \mathrm{~min} / \mathrm{dog}$ ) including tests measuring dog's inhibitory control, persistence, problem-solving strategy, and spatial problem-solving, and 
measured the dog's success in explosive search. In addition, we asked handlers opinion on their dogs general working performance.

Poor inhibitory or self-control has been shown to be associated with poorer cognitive performance both in dogs (Müller et al., 2015) and in humans (Duckworth 2015). Inhibitory control can also be observed as an activity in the same brain areas (frontal brain region) in humans and in dogs (Cook et al., 2016). In dogs, impulsivity has been shown to be a stable trait over time (Riemer et al., 2014). High impulsivity may predict behaviour problems in dogs (Wright et al., 2012) and increase the risk of externalizing disorders in humans. Individuals with good self-control can better regulate their behavioural, emotional, and attentional impulses compared to more impulsive individuals (Duckworth and Kern, 2011). Inhibitory control, a major part of self-control, is the ability to inhibit prepotent or impulsive, but ultimately counterproductive behaviour (de Ridder et al. 2011). In animals, inhibitory control is necessary, for example in cooperative hunting or when living as a subordinate member in a hierarchical group (Marshall-Pescini et al., 2015). The ability to know when to hold back, and when to join in cooperative group hunting, may be an important factor for the success of the hunt (Marshall-Pescini et al., 2015) . Inhibitory control has been measured in humans, as well as in many animal species, dogs included (Verbruggen, 2009; Maclean, Evan et al., 2014; Mayack and Naug, 2015). Impulsivity is a complex trait, which is divided into two overlapping concepts: impulsive choice and impulsive action (Diergaarde, 2008).

\section{Several tasks have been used to measure inhibitory control in animals. Majority of these tasks do} not correlate with each other, and those seem to reflect different aspects of inhibitory control (Brucks et al., 2017; Vernouillet et al., 2018) . In this study, we chose to measure impulsive action using the cylinder task (Bray et al., 2014) . In the cylinder task, the dogs are first trained to retrieve 
80 a reward out of an opaque cylinder, which is open on both ends. After several training trials, the

81 cylinder is made transparent, and the dogs could see the reward inside. To get access to the

82 reward they need to go to the sides of the cylinder and inhibit reaching for the reward directly (by

83 touching the glass). Cylinder task was chosen as we wanted a method, which has been used in

84 variety of species (Maclean et al., 2014), and most importantly, does not need lengthy training

85 period. Any task that requires lengthy pre-learning periods, may actually accidentally exclude the

86 most impulsive individuals, as the poor impulse control most likely slows down the learning

87 process. V-detour task has been suggested to measure spatial problem-solving ability, but also

88 inhibitory control (Marshall-Pescini et al., 2015) and this task was also included in the cognitive

89 test battery. In the V-detour-task, the dogs are required to walk away from the food reward and

90 first make a detour around a V-shaped fence to reach a reward.

91 Delayed gratification task has been often used in measuring inhibitory control. However, in dogs, it

92 has recently suggested to measure the persistency in focusing on the higher value/delayed reward

93 (Brucks et al., 2017). This test was also found to correlate negatively with tests measuring more

94 spontaneous motor inhibition and with impulsivity scores derived from same dogs (DIAS, Dog

95 Impulsivity Assessment Scale) (Brucks et al., 2017). It was also suggested to measure more

96 motivation than inhibitory control, and due to these above-mentioned factors, this task was left

97 out from the test battery.

99 In addition to good inhibitory control, traits most likely important for an explosive search dog are

100 the ability to work and solve problems independently and persistently. However, the dog also needs

101 to be guided and controlled during search, and thus complete independency, as a problem-solving 
102

103

104 117 persistence in dogs is lacking.

119 This study investigates whether the individual variation in the canine cognitive traits of inhibitory task.

strategy, might not be optimal. An "impossible" or "unsolvable task" has frequently been used with dogs to test the dog's tendency to seek help or look back towards humans (Miklósi et al., 2003). In this type of task, dogs are confronted with a box, which is easy to open at first, but which finally becomes impossible to open. Dogs vary a great deal in the main strategy they use to try to open the box; some are independent while others are quick to seek help from humans by gazing in their direction. The time spent gazing towards humans for help has been found to have a relatively large heritable component ( $\mathrm{h}^{2}$ 0.37) (Persson et al., 2015), and several loci through GWAS (Genome-Wide Association) studies have also been indicated for this behaviour (Persson et al., 2016). An independent problem-solving strategy has been interpreted as persistence in a recent study, where wolves were suggested to be more persistent compared to dogs when tackling an unsolvable task (Marshall-Pescini et al., 2017). Persistence is a trait that is highly associated with good self-control and with a conscientiousness personality trait in humans, especially in children (Caspi et al., 2005). Generally more studied in humans (Baer et al., 2015), persistence has only recently raised interest in canine studies (Dalal and Hall, 2019). Persistence is most likely a trait that varies tremendously between individuals as well as between dog breeds, yet a methodology for reliably testing control, persistence, problem-solving strategy, and spatial problem-solving correlate with work performance in Finnish police dogs specifically trained for explosive detection. Our hypothesis is that more persistent dogs with good inhibitory control perform better in the explosive detection

\section{Materials and methods}


A total of 24 trained and healthy Finnish police explosive search dogs participated in the study. The age of the dogs varied between 12 and 112 months (mean age 58 months), and they represented five breeds (eleven Belgian Shepherd Malinois, eight German Shepherds, three Labrador retrievers, one English springer spaniel and one Dutch Shepherd mix). Only two of the tested dogs were females. 18 dogs were dual purpose dogs (i.e dogs trained both for explosive detection and attack / protection) and only five dogs were single purpose dogs (trained only for explosive detection).

All of the dogs were first tested with a short cognitive test battery, which was conducted for 23 dogs at the Finnish Police Dog Training Center (Hämeenlinna), and for one dog in Vantaa. Police dog handlers also filled in a questionnaire on the dog's daily routines, performance in searching in actual work, arrival age, amount of weekly training, handler experience, and so forth. Finally, all dogs participated in annual qualification test to monitor each dog's explosive searching abilities.

\section{Behavioural test}

Each dog was assessed using a short cognitive test battery including a cylinder test, a V-detour and an unsolvable task. Food (sausage, $1.5 \mathrm{~cm} \times 1.5 \mathrm{~cm}$ ) was used as reward in each task. The dog was released into the test room, and was allowed freely to explore the room (approximately $5 \mathrm{~min}$ ) while the handler filled out the dog's information sheet. During the test, only the experimenter (KT), dog handler and a dog were present in the room.

\section{Behavioural test - Cylinder task}

Cylinder test was done according to Bray et al. (2014), however, the major difference in the methods was, that the dog saw the food entering the cylinder, both in the learning and in the test phases. In the learning phase, the dog is taught that the opaque cylinder contains food, and that the dog is able to get the food from the open end of the cylinder $(25 \mathrm{~cm}$ wide, with $20 \mathrm{~cm}$ opening) (Fig 1a). 
150 Dog is called by its name to get its attention, and food is shown to the dog, and then placed into the

151 cylinder. Experimenter then gives the permission to the handler to release the dog. In both the

152 learning and the test phase the dog is released from $180 \mathrm{~cm}$ distance from the cylinder, and the dog

153 sees the food entering the cylinder. The criteria for successful learning was 4 correct attempts

154 (taking food without touching the cylinder) out of 5 trials, where the maximum trials in the learning

155 phase was set to 15 . Only one dog (Malinois) did not pass the learning phase, and was thus excluded

156 from the analysis of cylinder test. After successful learning, the cylinder is made transparent, and

157 consequently, the dogs can see the reward inside (Fig b). In the actual test phase, the food was

158 placed inside the transparent cylinder ten consequent times, and the dog's success and errors were

159 counted. An error occurred when the dog attempted to reach the food directly through the

160 plexiglass (i.e. by touching the glass with either its paw(s) or nose), which is considered to reflect

161 poor inhibitory control. Success in this task varied from $0 \%$ (errors in all ten trials) to $100 \%$ (no errors 162 made in ten trials).

163

164 Behavioural test - V-detour

165 In the V detour task (Pongrácz, 2001), the dog was allowed to see when the food was placed

166 inside a V-shaped fence, into the narrow end of the V-detour. The length of both sides was 180

$167 \mathrm{~cm}$, and height of the fence was $69 \mathrm{~cm}$, and dog could see the food through the fence (Fig 1c). The

168 experimenter places the food by leaning over the fence (i.e. not going inside the fence).

169 Immediately after the placement of food inside the fence the dog was released from

170 approximately $40 \mathrm{~cm}$ distance from the narrow end of fence. The experimenter stands beside the

171 fence facing the fence (Fig $1 \mathrm{c}$ ). To successfully reach the food, the dog has to move further away

172 from the food, and make a detour successfully to reach the food. It has been suggested that

173 individuals with lower inhibitory control will be unable to fight their desire to head straight for the 
174 food, an unproductive choice that results in more time elapsing before they ultimately reach the

175 treat (Marshall-Pescini et al., 2015). The time it took for the dog to reach the food inside the V-

176 detour at the first attempt was measured (Bray et al., 2015), keeping the maximum time to three

177 minutes. There was only one trial for each dog.

178

Behavioural test - Impossible task

180 Finally, the dogs were tested with the impossible task paradigm, whereby the methodology is 181 followed according to previous studies (Miklósi et al., 2003). In this task, the dog is initially

182 confronted with an easy problem-solving task (removing a piece of sausage from a transparent 183 plastic box three times). In the first three trials, the lid of the box is not locked on its bottom, and 184 therefore is easy to push aside and reach the food. On the fourth occasion, the task looks similar, 185 but the lid of the box is now locked to the bottom, and impossible to open (Fig $1 \mathrm{~d}$ ). Also, the amount 186 of sausage inside is 10 pieces (in the training trials it is one piece) to ensure the motivation in the 187 task. During the subsequent two-minute period, three categories of behaviours were measured: 1) 188 the time that the dog spends manipulating the box with its nose or paw(s), (i.e. independent 189 strategy, previously interpreted as persistence in Marshall-Pescini et al. (2017) ; 2) the time spent 190 looking at the tester / handler OR looking back and forth between the tester / handler and the task

191 OR trying some other previously learned tasks, such as lying/sitting AND looking either tester / 192 handler or the box (i.e. human-dependent strategy); and 3) abandoning the task - sniffing the 193 ground, running around, exploring the room, not focusing on either the humans or the box (i.e. 194 giving up, negatively correlated with persistence). We calculated the seconds for each of the three 195 behaviours during the two-minute period, and the percentage of each three behaviours was 196 subsequently used in the analysis. 
198

199

200

\section{Qualification test}

Finally, all of the dogs participated in an annual explosive search test, which is an official test of Finnish Police to monitor each dog's explosive searching abilities. This qualification test was planned and evaluated by the teacher at the Finnish Police Dog Training Center (AT). This annual qualification test was conducted two months after the cognitive testing, and included 12 different explosives hidden in two large buildings (local school). The search test in these two locations was divided into two separate days. The searching situation and location was such that it would reflect as much as possible a real-life situation. The dog needed to find at least eight of the 12 explosives in order to continue working as an explosive detection dog. Success in the test was rated based on the number of found explosives (numerical value ranging from 0 to 12). Also "false positives" were recorded (false positive $=$ dog clearly indicates that it found an explosive, handler approves dog's signalling, but there is no explosive at that location).

\section{Questionnaire and data analysis}

Police dog handlers were asked to complete a questionnaire prior to the annual qualification test. The questionnaire included questions on the dog's daily routine, background, training, general working performance and behaviour (Attachment 1). Specifically, we were interested on how does the owner perceive the dog's working abilities (persistence, independence, ability to guide the dogs search, asking for help, and giving up). Also, we asked owner evaluation on the dog's sociability (friendliness), sudden aggressive behaviour, ability to calm down, and police-car related stress behaviour. Part of the questions in the questionnaire were derived and modified to suit for search task from an earlier published questionnaire (Wright et al., 2011; Tiira and Lohi, 2014) . 
221 We were interested to find out whether any of the measured variables in the behavioural test, or

222 in the questionnaire were associated with success in the explosive search test. We used

223 Spearman's Correlation Coefficient to analyse the correlation between success in the explosive

224 search task, the behavioural test variables and the questionnaire variables. Correction for multiple

225 testing was done using Benjamini-Hochberg procedure (Benjamini, 1995) using a critical value for

226 discovery rate as 0.10 . Corrected values are presented.

229 Results

231 A total of 23 dogs participated in the behavioural test battery, 25 into the qualification test and 23 232 handlers answered the questionnaire. The number of dogs that we had data from both behavioural 233 test battery and qualification test was 23 . However, one dog was left out from the analysis of the 234 cylinder task, due to not learning the first cylinder phase, thus leaving the sample size for the 235 cylinder task analysis $\mathrm{N}=22$. In addition, in the impossible task, six dogs broke the plastic box before $2362 \mathrm{~min}$ period ended, and thus reached the food. As we do not have the data from the whole $2 \mathrm{~min}$ 237 period, we felt that it was safe to exclude all these individuals from the later analysis of the 238 impossible task, thus leaving the sample size for the impossible task as 17 . The mean values for 239 variables investigated are presented in the Table 1. In two occasions at the qualification test, the 240 dogs' performance was interrupted by the handler or the judge (as the dog was unable to 241 concentrate on searching). These dogs were included in the analysis and the number of found explosives was set 0 for these dogs. 
Success in the cylinder task was the only factor that was significantly associated with success in the explosive search task. Dogs that had a high success rate in explosive detection had fewer errors in the cylinder task compared to dogs with a low success rate (Spearman's correlation coefficient $r_{s}=$ $0.466, P=0.033, N=22$, Fig. 2). The percentage that each dog spent on using different problemsolving strategies (independent $P=0.463$; human-dependent $P=0.975$; abandoning the task, giving up $P=0.556, N=17$ ), was not associated with the search task success. The search task success was not associated with the time taken to solve the $V$-detour $(P=0,681)$ either. The handler's assessment of the dog's general working abilities (persistence $P=0.80$, giving up $P=0.945$, controllable $P=0.846$, asking for help $P=0.059$ ) was not associated with success in explosive searching. However, the number of errors (false positives) in the explosive search task was associated with the handler's evaluation on the dog's persistence $\left(r_{s}=0.460, P=0.030, N=23\right)$, where dogs evaluated by their handlers as more persistent also made more errors in the explosive search task. Everyday routine variables (amount of training $P=0.419$, daily exercise $P=0.853$ ) or age $(P=0.151)$ were not associated with success in the search task.

\section{There were, however, several significant correlations between owner assessment of a dog's} working abilities and behavioural test variables. Those dogs that made more errors in the cylinder task tended to be evaluated by their handlers as giving up sooner in the explosive search $(r=-$ 0.446, $P=0.053, N=21)$, and these dogs also abandoned the impossible task box more easily $(r=-$ $0.518, P=0.040, N=16$ ), thus being less persistent. Dogs that abandoned the impossible task box for longer periods, were also estimated to give up easily during the search by their handlers ( $r=$ $0.515, \mathrm{P}=0.044, \mathrm{~N}=16$ ). Furthermore, the time that the dog spent trying to open the box (independent strategy) was positively associated with the handler-evaluated tendency to give up during the explosives search $(r=0.595, P=0.019, N=16)$. Moreover, human-associated strategy 
268

was negatively correlated with handler-evaluated tendency to give up during a search; humanoriented dogs were evaluated as less likely to give up $(r=-0.766, P=0.007, N=16)$.

Several handler-evaluated traits in the questionnaire were also correlated with each other. Dogs that were trained less (per week) were also evaluated to be more likely to give up during a search ( $r$ $=-0.710, P=0.004, N=23$ ). Dogs asking more help (handler-evaluated trait) had difficulties in calming down at home $(r=-0.448, P=0.037, N=23)$, were more likely to give up during search $(r=$ $0.428, P=0.048, N=23)$, and had more aggressive behaviour $(r=0.467, P=0.023, N=23)$. Dogs that were evaluated as being more persistent in search were also evaluated to be easily controlled during search $(r=0.576, P=0.01, N=23)$.

We also observed breed differences in the cylinder task; Malinois $(\mathrm{N}=10)$ made fewer errors compared to German Shepherds $(\mathrm{N}=6)$ (Mann-Whitney $U$ test, $\mathrm{P}=0.015)$. There was also a tendency for Malinois to experience better success in explosive detection (Mann-Whitney U test, P $=0.056$ ), and to do less errors (false positives) during the explosive search test (Mann-Whitney $U$ test, $\mathrm{P}=0.020)$. The datasets generated during and/or analyzed during the current study are available from the corresponding author upon request.

\section{Discussion}

Self-control has been among the most widely studied subjects in human social sciences in recent decades (Duckworth, 2015). In humans, good self-control is associated with lifelong benefits in several areas of life (Moffitt et al., 2011), but little is known about the performance of dogs with variable inhibitory control. We found that explosive detection dogs with good inhibitory control, 
292 (fewer errors in the cylinder task), were more successful in explosive detection, as expected, and 293 were also evaluated by their handlers as being more persistent in general when it came to search 294 tasks. This is the first time that an association has been found between working dog success and 295 inhibitory control with dogs. A recent study, that also investigated the association between cognitive 296 traits and explosive search success, did not find any association between the cylinder task and dog's 297 performance (MacLean and Hare, 2018). As a measure of dog's performance, the authors used 298 "various training and performance-related records" which were obtained from the dog trainers 299 (MacLean and Hare, 2018). Our measure of dog's working performance was resembling as closely 300 as possible the real-life situation of an explosive search dog. In general, we feel that the studies 301 investigating the working dog "performance" should have more accurate measures of performance, 302 that reflect the actual working success, and not the trainability of the dog. Moreover, there is most 303 likely a large difference between the different types of dogs (pet and police dogs) and different 304 breeds in the performance in the cylinder task. The average cylinder task success in our study with 305 police dogs was $66.8 \%$, while in the earlier studies with pet dogs, the average success was $82 \%$ 306 (Vernouillet et al., 2018) or even $95 \%$ (Marshall-Pescini et al., 2015) . In dogs, inhibitory control 307 most likely has a heritable component (Fadel et al., 2016) , but it is also context dependent and can 308 be affected by training and experience (Glady et al., 2012). Recent selection, as opposed to historical 309 selection, is pronounced in the behavioural profiles and personalities of contemporary dog breeds 310 (Svartberg, 2006). Many dog breeds are clearly divided into two separate breeding lines, whereby 311 in work-line breeding, the major breeding criterion is success in actual work (police, customs, 312 hunting etc.) or success in working dog competitions. Correspondingly, in show-line breeding, 313 appearance and success in dog shows are the most important criteria for breeding, and most pet 314 dogs belong to the show-line. Selection in working dog breeding lines has favoured impulsive 315 behaviour (Fadel et al., 2016), as well as hyperactivity (Foyer et al., 2014). An easily aroused dog, 
316 which behaves without hesitation, is most likely easier to train for tasks that demand risky

317 behaviour, such as protection or certain hunting tasks (e.g. cave hunting dogs, which must confront 318 a much larger animal in a confined space) (Brady et al., 2018). Hyperactive-impulsive dogs also seem 319 to have a higher reward responsiveness (Gerencser et al., 2018), which is a very useful trait in dog 320 training. The most popular police dog and working dog breeds (working line), Belgium Shepherd 321 Malinois and German Shepherd, may thus have a lower inhibitory control compared to pet dogs due 322 to selective breeding. Low inhibitory control is most likely very suitable for protection and attack, 323 however, may not be ideal for longer working tasks, which demand stamina and the ability to work 324 with a lower arousal level. Also, highly aroused dogs pant more than calm dogs, and as olfaction and 325 panting have an inverse relationship (Jenkins et al., 2018), panting due to high arousal may reduce 326 the ability to smell. Moreover, in an earlier study, the canine problem-solving ability was shown to 327 be worse in dogs, with low inhibitory control, compared with dogs with better inhibitory control 328 (Müller et al., 2015). Defects to inhibit impulsive actions may also worsen the ability to concentrate 329 on the odour related task in the qualification task.

331 Inhibitory control has been measured in humans (Duckworth and Kern, 2011) as well as in dogs

332 (Brucks et al., 2017) using various approaches (behavioural tests, questionnaires). In both species 333 the main finding has been that the different measures do not correlate with each other, and most 334 likely measure different aspects of inhibitory control. Inhibitory control appears to be a complex 335 trait, and as different tests seem to measure different aspects of this ability, practitioners have 336 been cautioned against using a single task as a measure of inhibitory control (Brucks et al., 2017) . 337 The V-detour, a task also suggested to measure inhibitory control, did not correlate in our study 338 with success in the explosive search task, nor with the cylinder test success. Lack of correlation 
339 between the V-detour and the cylinder task has also been observed before (Marshall-Pescini et al.,

340 2015). The reason for the lack of correlation between different tasks (and questionnaire(s)) might

341 be that those evaluate different inhibitory control abilities, and furthermore, are most likely

342 context specific (Vernouillet et al., 2018).

344 The cylinder task errors demonstrating counterproductive behaviour, such as the dog touching the 345 plexiglass in consecutive trials with their nose and paw(s) in order to access food, have recently been 346 interpreted as indicating persistence in dogs (Brucks et al., 2017). Our results do not support this; 347 dogs with fewer cylinder task errors were evaluated by their owners as being more persistent in real 348 working tasks, and these dogs were also less likely to abandon the impossible task box. Moreover, 349 these dogs had better success in the explosive search qualification test - a task that demands real 350 working persistence. The personality trait of conscientiousness in children associates with individual 351 differences in self-control (Caspi et al., 2005; Duckworth and Seligman, 2017). One of the traits in 352 highly conscientious children is persistence (Caspi et al., 2005), this suggesting that persistence in 353 animals also most likely correlates with good inhibitory control, as was also found in our study. Also, 354 one of the definitions of impulsivity is the lack of perseverance, which means the difficulty of 355 focusing on a task that may be boring or difficult (Roberts et al., 2011). The term persistence should 356 not be conflated with inflexibility in changing from unadaptive behaviour to more appropriate 357 behaviour, due to a high arousal state. We feel that this inflexibility in behaviour regulation is most 358 likely the explanation for high number of errors in dogs. Dogs with higher basal arousal level most 359 likely perform worse in a problem-solving task when arousal level is increased (Bray et al., 2015). 360 We suspect, that, in our study, the dogs with high number of errors in the cylinder task most likely 361 also have a higher basal arousal level. This, however, needs more research. 
363 Against our hypothesis, the dog's persistence, measured in the impossible task was not associated 364 with the success in the explosive search task. There are several possible explanations for this; (1) 365 either persistence is not an important trait for this task, (2) there is no variation in the persistence 366 in the study population, (3) the sample size in this task was too small $(N=17)$ or finally (4) the 367 impossible task's independent strategy does not measure persistence. We feel, that together with 368 the small sample size, the latter explanation might be the most likely one. The impossible task has 369 been used in several studies assessing dogs' problem-solving strategies, with human gazing being a 370 particular the focus of interest (Miklósi et al., 2003; Passalacua et al., 2013; Marshall-Pescini et al., 371 2017). Behaviour in this task can be divided into three main categories. First, the dog can 372 independently try to open the box (independent strategy). Second, the dog seeks for help either by 373 gazing at humans, looking back and forth between humans and the box, or doing a previously 374 learned task such as sitting, barking, and so on. All of these human-focused strategies were 375 combined in this study under a human-associated strategy, which is an active strategy whereby the 376 dog attempts to open the box by using human help. Finally, the dog can also choose to abandon the 377 box (i.e. by going away, or exploring the room). An independent strategy has recently been 378 interpreted as persistence and human-associated gazing as giving up (Marshall-Pescini et al., 2017). 379 Contradicting this hypothesis, in the present study the independent strategy was associated with 380 the tendency to give up searching (handler assessment) and, correspondingly, the human381 associated strategy was strongly correlated with less likelihood of giving up (handler assessment). 382 However, abandoning the box (not focusing on the box at all) was associated with the handler's 383 evaluation of dog's tendency to give up, and we suggest that this could be used in the future to 384 measure the likelihood of giving up in a task. The inability to switch from an unsuccessful strategy 385 in the impossible task (independent strategy) to an alternative one (seeking help from humans) may 
not actually reflect persistence, but inflexibility due to a highly aroused state. However, this calls for more research using a larger study population with more genetic variation (different breeds). The study population and its genetic variation (breeds) most likely has a large influence on results in canine cognitive studies, especially with small sample sizes.

We also found that the Malinois breed made fewer errors during the cylinder task, and these dogs also tended to have better success in the explosive search test. The data, however, was very small for each breed, and should therefore be treated with caution and replicated with a larger dataset.

This study, suggests that inhibitory control may be one important aspect to consider when selecting suitable dogs for explosive detection. Working dog success should be in the future be assessed using methods that reflect the actual working ability, and not trainability. Most typically canine cognitive studies focus on small group of dogs originating from several breeds, which is a major source of mixed results. Canine cognitive studies, which have objective cognitive test-data, and large sample sizes from different breeds are crucially needed.

\section{Acknowledgements}

We are most grateful for all the police dog handlers who participated in this study. This work was financially supported by the Police Dog Training Centre.

\section{Ethical approval}

All procedures performed in studies involving human participants were in accordance with the ethical standards of the institutional and/or national research committee and with the 1964 
409 Helsinki declaration and its later amendments or comparable ethical standards. All applicable

410 international, national, and/or institutional guidelines for the use of animals were followed.

412 Declaration of interest

413 This work (for K.Tiira) was financially supported by the Police Dog Training Centre. Authors have 414 no competing interests to declare.

415

416

417

418

419

420

421

422

423

Bray, E., MacLean, E., Hare, B., 2015. Increasing arousal enhances inhibitory control in calm but

References

Baer, J., Schreck, M., Althoff, R.R., Rettew, D., Harder, V., Ayer, L., Albaugh, M., Crehan, E., KunySlock, A., Hudziak, J.J., 2015. Child Temperament, Maternal Parenting Behavior, and Child Social Functioning. Journal of Child and Family Studies 24, 1152-1162 doi: 10.1007/s10826-014-9924-5.

Benjamini, Y., 1995. Controlling the False Discovery Rate: A Practical and Powerful Approach to Multiple Testing. Journal of the Royal Statistical Society.Series B (Methodological) 57, 289-300.

Brady, K., Cracknell, N., Zulch, H., Mills, D.S., 2018. Factors associated with long-term success in working police dogs. Appl. Anim. Behav. Sci. 207, 67-72 doi: 10.1016/j.applanim.2018.07.003. not excitable dogs. Anim Cogn 18, 1317-1329 doi: 10.1007/s10071-015-0901-1. 
431 Bray, E., MacLean, E., Hare, B., 2014. Context specificity of inhibitory control in dogs. Anim Cogn 432 17, 15-31 doi: 10.1007/s10071-013-0633-z.

433 Bray, E., Sammel, M., Seyfarth, R., Serpell, J., Cheney, D., 2017. Temperament and problem solving 434 in a population of adolescent guide dogs. Anim. Cogn. 20, 923-939 doi: 10.1007/s10071-017-11124358 [doi].

436 Brucks, D., Marshall-Pescini, S., Wallis, L.J., Huber, L., Range, F., 2017. Measures of Dogs' Inhibitory 437 Control Abilities Do Not Correlate across Tasks. Frontiers in Psychology 8 doi: $438 \quad$ 10.3389/fpsyg.2017.00849.

439 Caspi, A., Roberts, B.W., Shiner, R.L., 2005. Personality development: stability and change. Annual 440 review of psychology 56, 453-484 doi: 10.1146/annurev.psych.55.090902.141913.

441 Cook, P.F., Prichard, A., Spivak, M., Berns, G.S., 2016. Awake canine fMRI predicts dogs' preference 442 for praise vs food. Social cognitive and affective neuroscience 11, 1853.

443 Corr, P., 2010. Individual Differences in Cognition: in Search of a General Model of Behaviour 444 Control, in: Anonymous Handbook of Individual Differences in Cognition. First ed. Springer New 445 York, New York, NY, pp. 3-26.

446 Dalal, S., Hall, N.J., 2019. Behavioral Persistence is Associated with Poorer Olfactory Discrimination 447 Learning in Domestic Dogs. Behav. Processes, 64-71.

448 Diergaarde, L., 2008. Impulsive Choice and Impulsive Action Predict Vulnerability to Distinct Stages 449 of Nicotine Seeking in Rats. Biol. Psychiatry 63, 301-308. 
450 Duckworth, A., Seligman, M., 2017. The Science and Practice of Self-Control. Perspectives on 451 Psychological Science 12, 715-718 doi: 10.1177/1745691617690880.

452 Duckworth, A., 2015. The significance of self-control. PNAS 108, 2639-2640 doi:

$453 \quad 10.1073 /$ pnas.1019725108.

454 Duckworth, A.L., Kern, M.L., 2011. A meta-analysis of the convergent validity of self-control 455 measures. Journal of Research in Personality 45, 259-268 doi: 10.1016/j.jrp.2011.02.004.

456 Ennik, I., Liinamo, A., Leighton, E., van Arendonk, J., 2006. Suitability for field service in 4 breeds of 457 guide dogs. Journal of Veterinary Behavior: Clinical Applications and Research 1, 67-74 doi: $458 \quad$ 10.1016/j.jveb.2006.06.004.

459 Fadel, F.R., Driscoll, P., Pilot, M., Wright, H., Zulch, H., Mills, D., 2016. Differences in Trait 460 Impulsivity Indicate Diversification of Dog Breeds into Working and Show Lines. Scientific reports $461 \quad 6,22162$ doi: 10.1038/srep22162.

462 Foyer, P., Bjällerhag, N., Wilsson, E., Jensen, P., 2014. Behaviour and experiences of dogs during 463 the first year of life predict the outcome in a later temperament test. Appl. Anim. Behav. Sci. 155, 464 93-100 doi: 10.1016/j.applanim.2014.03.006.

465 Gerencser, L., Bunford, N., Moesta, A., Miklosi, A., 2018. Development and validation of the 466 Canine Reward Responsiveness Scale -Examining individual differences in reward responsiveness 467 of the domestic dog. Sci. Rep. 8, 1 doi: 10.1038/s41598-018-22605-1 [doi].

468 Glady, Y., Genty, É, Roeder, J., 2012. Brown Lemurs (Eulemur fulvus) Can Master the Qualitative 469 Version of the Reverse-Reward Contingency. PLoS One 7, e48378 doi:

$470 \quad 10.1371 /$ journal.pone.0048378. 
471 Griffin, A.S., Guillette, L.M., Healy, S.D., 2015. Cognition and personality: an analysis of an 472 emerging field. Trends in ecology \& evolution 30, 207-214 doi: 10.1016/j.tree.2015.01.012.

473 Jenkins, E.K., Dechant, M.T., Perry, E.B., 2018. When the Nose Doesn't Know: Canine Olfactory 474 Function Associated With Health, Management, and Potential Links to Microbiota. Frontiers in 475 Veterinary Science 5, 56.

MacLean, E., Hare, B., 2018. Enhanced Selection of Assistance and Explosive Detection Dogs Using Cognitive Measures. Frontiers in Veterinary Science 5.

Maclean, E., Hare, B., Nunn, C.L., Addessi, E., Amici, F., Anderson, R.C., Aureli, F., Baker, J.M., Bania, A.E., Barnard, A.M., Boogert, N.J., Brannon, E.M., Bray, E.E., Bray, J., Brent, L.J.N., Burkart, J.M., Call, J., Cantlon, J.F., Cheke, L.G., Clayton, N.S., Delgado, M.M., Divincenti, L.J., Fujita, K., Herrmann, E., Hiramatsu, C., Jacobs, L.F., Jordan, K.E., Laude, J.R., Leimgruber, K.L., Messer, E.J.E., Moura, Antonio C De A, Ostojić, L., Picard, A., Platt, M.L., Plotnik, J.M., Range, F., Reader, S.M., Reddy, R.B., Sandel, A.A., Santos, L.R., Schumann, K., Seed, A.M., Sewall, K.B., Shaw, R.C., Slocombe, K.E., Su, Y., Takimoto, A., Tan, J., Tao, R., Van Schaik, C.P., Virányi, Z., Visalberghi, E., Wade, J.C., Watanabe, A., Widness, J., Young, J.K., Zentall, T.R., Zhao, Y., 2014. The evolution of self-control. Proceedings of the National Academy of Sciences 111, E2148 doi: 10.1073/pnas.1323533111.

Marshall-Pescini, S., Rao, A., Virányi, Z., Range, F., 2017. The role of domestication and experience in 'looking back' towards humans in an unsolvable task. Scientific Reports (Nature Publisher Group) 7, 46636 doi: 10.1038/srep46636.

Marshall-Pescini, S., Virányi, Z., Range, F., 2015. The effect of domestication on inhibitory control: wolves and dogs compared. PLoS ONE 10, e0118469. doi:10.1371/journal.pone.0118469. 
493

494 495 496 497 498

Mayack, C., Naug, D., 2015. Starving honeybees lose self-control. Research .

Miklósi, Á, Kubinyi, E., Topál, J., Gácsi, M., Virányi, Z., Csányi, V., 2003. A Simple Reason for a Big Difference:Wolves Do Not Look Back at Humans, but Dogs Do. Current Biology 13, 763-766 doi: 10.1016/S0960-9822(03)00263-X.

Moffitt, T.E., Arseneault, L., Belsky, D., Dickson, N., Hancox, R.J., Harrington, H., Houts, R., Poulton, R., Roberts, B.W., Ross, S., Sears, M.R., Thomson, W.M., Caspi, A., 2011. A gradient of childhood self-control predicts health, wealth, and public safety. Proc. Natl. Acad. Sci. U. S. A. 108, 2693.

Müller, C., Riemer, S., Viranyi, Z., Huber, L., Ranhe, F., 2015. Inhibitory Control, but Not Prolonged Object Related Experience Appears to Affect Physical Problem Solving Performance of Pet Dogs2016.

Passalacua, S., Marshall-Pescini, S., Merola, I., Palestrini, C., Prato Previde, E., 2013. Different problem-solving strategies in dogs diagnosed with anxiety-related disorders and control dogs in an unsolvable task paradigm. Applied Animal Behaviour Science 147, 139-148.

Persson, M., Roth, L., Johnsson, M., Wright, D., Jensen, P., 2015. Human-directed social behaviour in dogs shows significant heritability. Genes, Brain and Behavior 14, 337-344 doi: 10.1111/gbb.12194.

Persson, M., Wright, D., Roth, L., Batakis, P., Jensen, P., 2016. Genomic Regions Associated With Interspecies Communication in Dogs Contain Genes Related to Human Social Disorders. Scientific Reports 6, 33439 doi: 10.1038/srep33439.

Pongrácz, P., 2001. Social learning in dogs: the effect of a human demonstrator on the performance of dogs in a detour task. Anim. Behav. 62, 1109-1117. 
514 Riemer, S., Mills, D., Wright, H., 2014. Impulsive for life? The nature of long-term impulsivity in 515 domestic dogs. Anim Cogn 17, 815-819 doi: 10.1007/s10071-013-0701-4.

516 Roberts, W., Fillmore, M.T., Milich, R., 2011. Linking impulsivity and inhibitory control using

517 manual and oculomotor response inhibition tasks. Acta Psychologica 138, 419-428 doi:

$518 \quad$ 10.1016/j.actpsy.2011.09.002.

519 Sinn, D.L., Gosling, S.D., Hilliard, S., 2010. Personality and performance in military working dogs:

520 Reliability and predictive validity of behavioral tests. Applied Animal Behaviour Science 127, 51-65

521 doi: 10.1016/j.applanim.2010.08.007.

522 Svartberg, K., 2006. Breed-typical behaviour in dogs-Historical remnants or recent constructs?

523 Appl. Anim. Behav. Sci. 96, 293-313.

524 Tiira, K., Lohi, H., 2014. Reliability and validity of a questionnaire survey in canine anxiety research.

525 Appl. Anim. Behav. Sci. 155.

526 Tomkins, L.M., Thomson, P.C., McGreevy, P.D., 2011. Behavioral and physiological predictors of 527 guide dog success. Journal of Veterinary Behavior: Clinical Applications and Research 6, 178-187 528 doi: 10.1016/j.jveb.2010.12.002.

529 Verbruggen, F., 2009. Models of response inhibition in the stop-signal and stop-change paradigms. 530 Neurosci. Biobehav. Rev. 33, 647-661.

531 Vernouillet, A., Stiles, L., Andrew McCausland, J., Kelly, D., 2018. Individual performance across 532 motoric self-regulation tasks are not correlated for pet dogs. Learning \& Behavior 46, 522-536 doi: 533 10.3758/s13420-018-0354-x. 
534 Wilsson, E., Sundgren, P., 1998. Behaviour test for eight-week old puppies-heritabilities of tested

535 behaviour traits and its correspondence to later behaviour. Applied Animal Behaviour Science 58,

536 151-162 doi: 10.1016/S0168-1591(97)00093-2.

537 Wright, H.F., Mills, D.S., Pollux, P.M.J., 2011. Development and Validation of a Psychometric Tool

538 forAssessing Impulsivity in the Domestic Dog ( Canis familiaris). Int J Comp Psychol 24, 210-225.

539 Wright, H.F., Mills, D.S., Pollux, P.M.J., 2012. Behavioural and physiological correlates of impulsivity

540 in the domestic dog ( Canis familiaris). Physiology \& Behavior 105, 676-682 doi:

$541 \quad$ 10.1016/j.physbeh.2011.09.019. 
543 Table 1. Descriptive statistics (sample size, mean and standard deviation) of the variables in explosive

544 qualification task, behavioural test and questionnaire.

545

\begin{tabular}{|c|c|c|c|}
\hline Variable & $\mathbf{N}$ & Mean & SD \\
\hline Found explosives (qualification task) & 25 & 8.40 & 2.86 \\
\hline False positives (qualification task) & 25 & 1.36 & 1.22 \\
\hline Cylinder, \% (Behavioural test) & 22 & 66.80 & 23.10 \\
\hline V detour, s (Behavioural test) & 23 & 31.26 & 39.80 \\
\hline Independent strategy, \%(Impossible task, behavioural test) & 17 & 45.08 & 28.65 \\
\hline $\begin{array}{l}\text { Owner dependent strategy, \% (Impossible task, } \\
\text { behavioural test) }\end{array}$ & 17 & 43.79 & 33.10 \\
\hline Abandoning the box \%(Impossible task, behavioural test) & 17 & 11.03 & 19.79 \\
\hline Dogs persistency at work (Questionnaire, 1-5) & 23 & 3.74 & 0.69 \\
\hline Dogs tendency to ask for help (Questionnaire, 1-5) & 23 & 2.61 & 1.08 \\
\hline Dogs tendency to give up (Questionnaire, 1-5) & 23 & 2.30 & 1.15 \\
\hline $\begin{array}{l}\text { How easy it is to control the dog during search } \\
\text { (Questionnaire, 1-5) }\end{array}$ & 23 & 4.10 & 0.79 \\
\hline Friendliness (Questionnaire, 1-5) & 23 & 3.57 & 1.24 \\
\hline Ability to calm down (Questionnaire, 1-5) & 23 & 4.30 & 1.11 \\
\hline Stress in the police car (Questionnaire, 1-5) & 23 & 2.95 & 1.63 \\
\hline Sudden aggression (Questionnaire, 1-5) & 21 & 2.00 & 1.21 \\
\hline
\end{tabular}


548 Fig 1. a) Non-transparrent (learning phase) and b) transparrent cylinder (test phase) measuring 549 inhibitory control, and the testers position during in the task, c) V-detour and d) closed impossible 550 task box, at the second phase of the test.

551 Fig 2. Scatter plot showing the association between cylinder task success (\%), which measures self552 control, and the number of explosives detected by the dogs during the search task ( $N=22$, two dots 553 are overlapping with other dots). 